\title{
INFECCIÓN NATURAL DE LUTZOMYIA CRUCIATA (DIPTERA: PSYCHODIDAE, PHLEBOTOMINAE) CON WOLBACHIA EN CAFETALES DE CHIAPAS, MÉXICO
}

\section{OSCAR MIKERY-PACHECO, ${ }^{1}$ CARLOS MARINA-FERNÁNDEZ, ${ }^{4}$ SERgio IBÁÑEZ-BERNAL, ${ }^{3}$ DANIEL SÁNCHEZ-GUILLEN ${ }^{2}$ y Alfredo CASTILLO-VERA ${ }^{2}$}

${ }^{1}$ Universidad Autónoma de Chiapas. Centro de Biociencias. Carretera a Puerto Madero km 2.0, 30700 Tapachula, Chiapas, México.

${ }^{2}$ El Colegio de la Frontera Sur - Tapachula, Chiapas, México, Km 2.5 carretera Antiguo Aeropuerto, 30700 Tapachula, Chiapas, México.

${ }^{3}$ Instituto de Ecología, A.C., Red Ambiente y Sustentabilidad, Carretera Antigua a Coatepec No. 351, El Haya, Xalapa, 91070, Veracruz, México.

${ }^{4}$ Centro Regional de Investigación en Salud Pública. 19 Poniente Esq. $4^{\mathrm{a}}$. Ave. Norte, Col. Centro, 30700 Tapachula, Chiapas, México,

Mikery-Pacheco, O., C. Marina-Fernández, S. Ibáñez-Bernal, S. Sánchez-Guillen \& A. CastilloVera. 2012. Infección natural de Lutzomyia cruciata (Diptera: Psychodidae, Phlebotominae) con Wolbachia en cafetales de Chiapas, México. Acta Zoológica Mexicana (n. s.), 28(2): 401-413.

RESUMEN. Se muestran los resultados de la búsqueda de Leishmania y Wolbachia mediante PCR, en flebotominos recolectados durante 2011 (febrero-marzo) en cafetales de cuatro localidades ubicadas dentro de un transecto altitudinal en la región del Soconusco, Chiapas, México. Las especies Lutzomyia texana y $\mathrm{Lu}$. carpenteri son documentadas por primera vez para el estado de Chiapas. Wolbachia fue detectada en una hembra de Lutzomyia cruciata capturada en la Finca Guadalupe Sajú. Todas las muestras fueron negativas a Leishmania.

Palabras clave: Flebotominos, enfermedades transmitidas por vector, ecología, control biológico.

Mikery-Pacheco, O., C. Marina-Fernández, S. Ibáñez-Bernal, S. Sánchez-Guillen \& A. CastilloVera. 2012. Natural infection of Lutzomyia cruciata (Diptera: Psychodidae, Phlebotominae) with Wolbachia in coffee plantations from Chiapas, Mexico. Acta Zoológica Mexicana (n. s.), 28(2): 401-413.

ABSTRACT. Using PCR-based tools, we show the results of screening for Leishmania and Wolbachia in a phlebotomine sand flies collected during 2011 (February-march) in coffee plantations of four localities along an altitudinal transect in the Soconusco region, Chiapas, Mexico. The species Lutzomyia texana and $\mathrm{Lu}$. carpenteri are documented for the first time for the state of Chiapas. Wolbachia was

Recibido: 27/10/2011; aceptado: 06/02/2012. 
detected in a female of Lutzomyia cruciata captured in the Guadalupe Saju farm. All samples were negative to Leishmania.

Keywords: phlebotomine, sand flies, vector-borne diseases, ecology, biological control.

\section{INTRODUCCIÓN}

Las leishmaniasis son un grupo de enfermedades infecciosas causadas por protozoarios del género Leishmania (Kinetoplastida: Trypanosomatidae). A este género pertenecen veinte especies patógenas para el humano (Ashford 2000). La transmisión de estos parásitos ocurre por la picadura de insectos del género Lutzomyia (sensu lato) y Phlebotomus (Diptera: Psychodidae). Esta enfermedad es considerada como emergente y sin control por el TDR (Tropical Diseases Research), y se presenta en América, Europa, África y Asia (Remme et al. 2002). Se estiman alrededor de 350 millones de personas viviendo en zonas de riesgo, con dos millones de nuevos casos por año (WHO 2010).

Se han registrado casos de leishmaniasis en 98 países, incluido México. Su presencia ha sido registrada en 22 estados de la República Mexicana, con una mayor prevalencia de leishmaniasis cutánea (LC) y visceral (LV) en el estado de Chiapas (Sánchez-Tejeda et al. 2001, Becker et al. 2005). La LC en Chiapas afecta básicamente a la población humana de zonas selváticas y agrícolas, siendo considerada una enfermedad ocupacional (Becker et al. 2005, Chongo \& García 2010). La transmisión de Leishmania ha sido documentada en plantaciones de café, en México y otros países (Alexander et al. 2001, Alexander et al. 2002, Sanchez-Tejeda et al. 2001, Scorza et al. 1985). En México se han registrado 44 especies de flebotominos y una especie fósil (Young \& Duncan 1994, Ibáñez-Bernal et al. 2004, 2006, 2010, 2011), 25 de ellas en Chiapas. Entre las especies de importancia médica registradas en México se encuentran Lu. cruciata (Coquillett), Lu. ovallesi (Ortíz), Lu. longipalpis (Lutz \& Neiva), Lu. olmeca olmeca (Vargas \& Díaz-Nájera), Lu. evansi (Nuñez-Tovar) y Lu. shannoni (Dyar) (Ibáñez-Bernal et al. 2004, Sánchez-García et al. 2010).

Las poblaciones de estos vectores son combatidas principalmente con insecticidas sintéticos (piretroides y clorofluazurón como inhibidor del crecimiento), aunque no en México. También se han planteado alternativas biológicas (bacterias, síntesis de la feromona sexual, hongos y plantas tóxicas), como complemento para la destrucción de criaderos (Alexander \& Maroli 2003), que aún no están incluidas en la Norma Oficial Mexicana. El control genético es una alternativa muy prometedora para disminuir la población de vectores, mediante la distribución de organismos con modificaciones genéticas que intervengan en la transmisión de agentes patógenos (Sinkins \& Godfray 2004). Wolbachia es una bacteria intracelular obligada perteneciente a las $\alpha$-proteobacterias (Rickettsia), que es heredada maternalmente y se le encuentra en el tejido reproductivo de insectos (Werren 1997, Werren et al. 2008). Esta bacteria produce anormalidades reproductivas en el huésped, como partenogénesis, muerte 
de la progenie macho, feminización e incompatibilidad citoplásmica (IC) (Werren et al. 2008), con una presencia estimada en el 65\% de las especies de insectos (Hilgenboecker et al. 2008). La liberación de Aedes aegypti ( = Stegomyia aegypti) (L.) transformada con la cepa wMel de Wolbachia, bloquea la capacidad de las poblaciones silvestres para transmitir el dengue serotipo 2 (DENV-2) (Hoffmann et al. 2011, Walker et al. 2011). Los fenotipos modificados se propagan rápidamente en las poblaciones naturales de mosquitos, lo que ha despertado el interés en este microorganismo para usarlo en el control de insectos vectores de enfermedades e insectos plaga. El tamizaje de Wolbachia en flebotominos se ha aplicado a 38 especies en el mundo, ninguna procedente de México, y sólo se ha registrado infección en ocho especies (Azpurua et al. 2010, Cui et al. 1999, Ono et al. 2001). La detección de poblaciones infectadas en México podría incentivar el uso de este microorganismo en estrategias de control vectorial en un futuro próximo. El presente trabajo analizó la presencia de Leishmania spp. y Wolbachia en poblaciones silvestres de flebotominos en cafetales del Soconusco, Chiapas.

\section{MATERIALES Y MÉTODOS}

Recolección e identificación de flebotominos. Los insectos fueron capturados en cuatro localidades, ubicadas dentro de un transecto altitudinal de la zona cafetalera del Soconusco, Chiapas, durante los meses de febrero y marzo de 2011. Las localidades fueron: Teotihuacán del Valle (N1459’59.7'’ O092¹4'50.5'’, 434 msnm), Argovia (N1507'28.7' ' O092¹7'41.2', 608 msnm), San José Nexapa (N1503'46.2'” O092¹5'29.6”, 662 msnm) y Guadalupe Sajú (N1509'18.8', O092¹7’03.0’”, $1007 \mathrm{msnm}$ ) (Fig. 1). La distancia entre sitios fue mayor a $5 \mathrm{~km}$, para asegurar que los especímenes recolectados provinieran de diferentes poblaciones, ya que el rango de dispersión de los flebótomos es menor a esta distancia (Alexander 1987). Dos métodos de captura fueron aplicados por localidad durante tres noches consecutivas (7:00-8:00 p.m.). Cada noche, ocho trampas de luz blanca "CDC" (Model 512; John W. Hock Co., Gainesville, FL, EE.UU.) fueron suspendidas a $50 \mathrm{~cm}$ del suelo y separadas entre sí por $25 \mathrm{~m}$, en un transecto lineal dentro de cada localidad (Sudia \& Chamberlain 1962). Adicionalmente, una trampa Shannon con cebo humano en el interior fue instalada alejada 100m de las trampas de luz (Shannon 1939).

Los especímenes recolectados en cada sitio fueron depositados en frascos de vidrio con etanol al $96 \%$, etiquetados y almacenados a $-20^{\circ} \mathrm{C}$, hasta el momento de su montaje. El 10\% de los insectos capturados en cada localidad fueron preparados de cuerpo completo en laminillas para su identificación taxonómica mediante características morfológicas tradicionales. El resto de los insectos fueron decapitados y sus cabezas montadas en laminillas para identificación de las especies; la parte restante del cuerpo del insecto fue usada para la extracción del ADN del vector y de ambos 


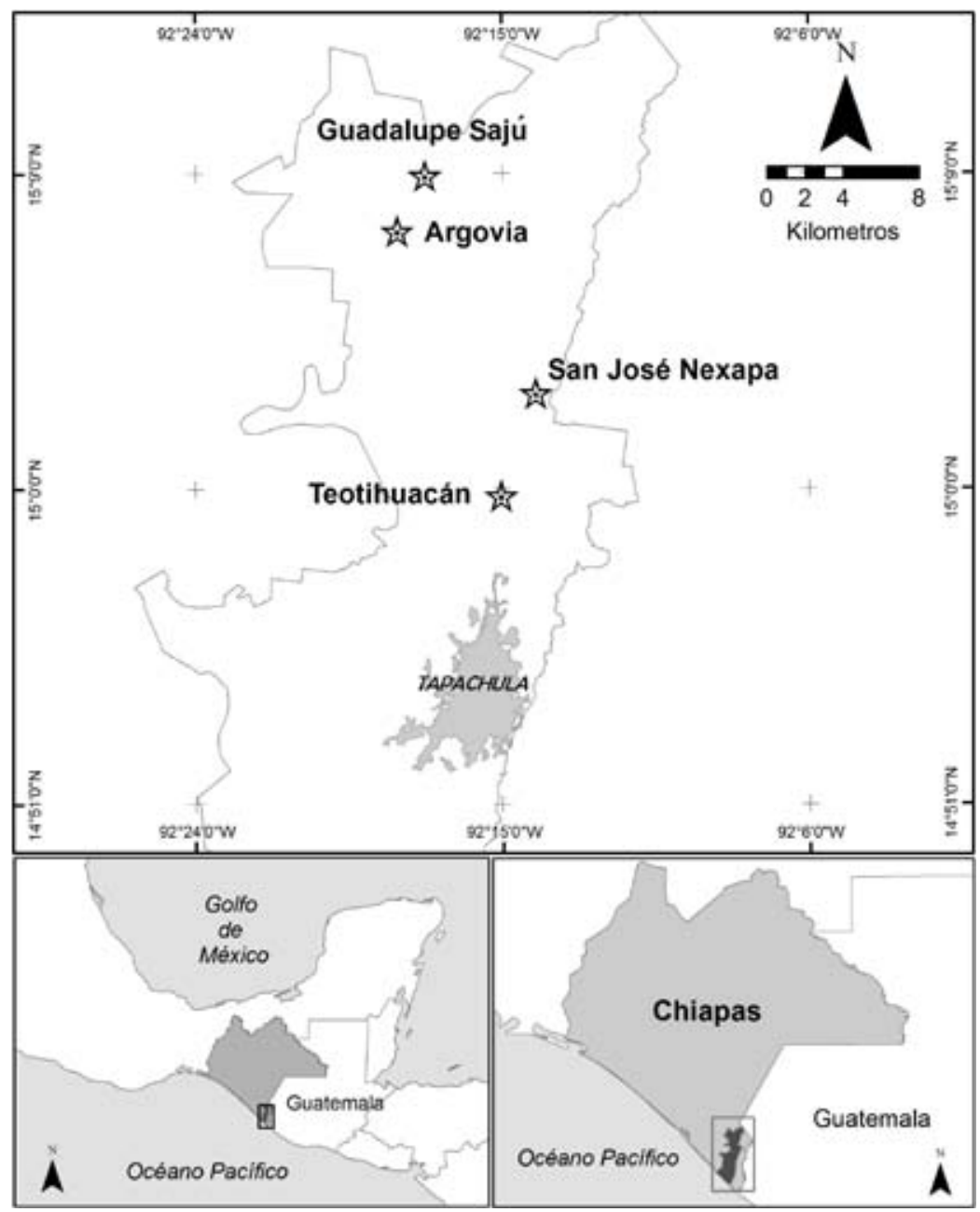

Figura 1. Ubicación geográfica de las localidades ( ) donde se capturó a los flebotominos.

microorganismos (Wolbachia y Leishmania). Los insectos enteros y las cabezas fueron procesados siguiendo el método descrito por Ibáñez-Bernal (2005a) para la elaboración de preparaciones permanentes en resina Euparal ${ }^{\circledR}$ (Bioquip Products, CA, EE.UU.) e identificados morfológicamente usando las claves de Young \& Duncan (1994) e Ibáñez-Bernal (1999, 2001, 2002, 2003, 2005a y b). Los abdómenes fueron colocados en tubos Eppendorf de $200 \mu \mathrm{L}$ (DNAsa/RNAsa free, Axygen, Inc., CA, EE.UU.) conteniendo $100 \mu \mathrm{L}$ de etanol $96 \%$ y almacenados a $-20^{\circ} \mathrm{C}$ hasta la extracción de ADN. Para este estudio no fue necesario utilizar el tórax de los insectos. 
Extracción de ADN. Las extracciones de ADN del vector, Leishmania y Wolbachia se hicieron en ejemplares hembra de flebotominos usando la técnica HotSHOT descrita por Montero-Pau et al. (2008). El abdomen de cada individuo fue transferido a un tubo Eppendorf de $0.2 \mathrm{~mL}$ conteniendo $50 \mu \mathrm{L}$ de solución tampón alcalina, para posteriormente ser triturado con puntas para pipeta previamente esterilizadas. Las muestras fueron incubadas a $95^{\circ} \mathrm{C}$ por 30 min y almacenadas en hielo por 3-4 min; después se le agregó $50 \mu \mathrm{L}$ de solución tampón neutralizante a cada tubo, finalmente fueron agitadas y ligeramente centrifugadas. Las muestras se almacenaron a $-20{ }^{\circ} \mathrm{C}$ hasta su posterior uso en las reacciones de PCR. Se hicieron grupos de ADN de 5 individuos por localidad para las reacciones.

Amplificación de fragmentos de ADN por PCR. Se mezcló 10uL del extracto de ADN de cada espécimen en un tubo Eppendorf de 200uL. En caso de un grupo positivo se analizó el ADN individual de cada uno de los especímenes que se mezclaron.

Para analizar la presencia de Leishmania spp., las muestras fueron tamizadas usando los iniciadores L.MC-1S y L.MC-1R específicos para la secuencia conservada dentro del ADN del minicírculo del cinetoplasto de Leishmania (Kato et al. 2005). El tamizaje para Wolbachia se realizo usando los iniciadores específicos del gen 16S ADNr (O’Neill et al. 1992) y wsp (Zhou et al. 1998) para detectar únicamente secuencias de Wolbachia. Los iniciadores 12SAI y 12SBI específicos para secuencias mitocondriales de insectos fueron usadas como control de calidad de cada extracción de ADN (O’Neill et al. 1992).

La mezcla de reacción usada en el tamizaje de Wolbachia y ADN del insecto se llevó a un volumen final de $10 \mu \mathrm{L}$, conteniendo solución tampón de PCR $1 \mathrm{X}$, dNTP, Taq polimerasa (REDTaq ${ }^{\circledR}$ SuperPak ${ }^{\mathrm{TM}}$ DNA Polymerase, Sigma-Aldrich, MO, U.S.A.), $1.0 \mu \mathrm{L}$ de ADN, $0.2 \mu \mathrm{L}$ del iniciador (16S ADNr, wsp o 12S mtADNr). La mezcla de reacción usada para el tamizaje de infección de Leishmania fue llevada a un volumen final de $10 \mu \mathrm{L}$ conteniendo Go Taq qPCR Master Mix 1X (PROMEGA), $0.4 \mathrm{uL}$ de cada iniciador y $1 \mu \mathrm{L}$ de ADN muestra. Los iniciadores usados en este estudio fueron sintetizados por Eurofins MWG Operon (Huntsville, Alabama 35805, EE.UU.).

El control positivo para Leishmania provino de un ratón infectado con Leishmania mexicana, y el control positivo de Wolbachia se obtuvo de Culex quinquefasciatus el cual es conocido por ser portador de Wolbachia (Glaser \& Meola 2010). Los mosquitos y el ADN de Leishmania fueron proporcionados por el Centro Regional de Investigación en Salud Pública (CRISP) Tapachula, Chiapas. Se uso agua destilada en sustitución de ADN como control negativo.

El perfil de temperatura aplicado a los iniciadores de Leishmania fue: $95{ }^{\circ} \mathrm{C}$ por 2 min, 30 ciclos a $95{ }^{\circ} \mathrm{C}$ por $1 \mathrm{~min}, 55^{\circ} \mathrm{C}$ por $1 \mathrm{~min}, 7{ }^{\circ} \mathrm{C}$ por 1 min y una extensión final de $72{ }^{\circ} \mathrm{C}$ por $10 \mathrm{~min}$. El protocolo de amplificación para los iniciadores $16 \mathrm{~S}$ 
ADNr y $12 \mathrm{~S}$ mtADNr, fue: $94{ }^{\circ} \mathrm{C}$ por 2 min, 37 ciclos a $94^{\circ} \mathrm{C}$ por $30 \mathrm{seg}, 55^{\circ} \mathrm{C}$ por $45 \mathrm{seg}, 72{ }^{\circ} \mathrm{C}$ por $1: 30 \mathrm{~min}$ y una extensión final de $72{ }^{\circ} \mathrm{C}$ por $10 \mathrm{~min}$. El perfil de temperatura para los iniciadores wsp fue: $94{ }^{\circ} \mathrm{C}$ por $1 \mathrm{~min}, 55^{\circ} \mathrm{C}$ por $1 \mathrm{~min}$ y $72{ }^{\circ} \mathrm{C}$ por $1 \mathrm{~min}$ durante 35 ciclos. Las amplificaciones se realizaron en un termociclador modelo C1000 ${ }^{\mathrm{TM}}$ (BIO-RAD). A los productos de PCR se les agregó SYBR ${ }^{\circledR}$ Green I nucleic acid gel stain $2 \times$ (SIGMA-ALDRICH) por $30 \mathrm{~min}$ y fueron corridos en gel de agarosa al 2\% con un marcador molecular de 100-1000 pb (Molecular PCR Low Ladder Marker Set, SIGMA-ALDRICH, Inc. S.L. U.S.A.), para determinar la presencia y tamaño del ADN amplificado. Los geles fueron observados y fotografiados bajo luz UV en un transiluminador (KODAK Gel Logic 200, Imaging System).

\section{RESULTADOS}

Recolección e identificación de flebotominos. Un total de 237 individuos fueron capturados en esta región cafetalera del sureste de México (Cuadro 1), en las localidades Teotihuacán del Valle (8.3\%), Argovia (28.2\%), San José Nexapa (24.3\%) y Guadalupe Sajú (39.3\%). El 71.4\% de las capturas fueron obtenidas con las trampas Shannon, el resto fue capturado con trampas CDC. El análisis morfológico realizado al 94.1\% del total de ejemplares permitió reconocer cinco especies pertenecientes al género Lutzomyia (sensu Young \& Duncan, 1994) el resto no logró ser identificado debido a su mal estado de preservación. Los 206 individuos identificados pertenecen a las especies Lu. ovallesi (Ortiz 1952) (0.5\%), Lu. shannoni (Dyar 1929) (0.9\%), Lutzomyia texana (Dampf 1938) (0.5\%), Lutzomyia carpenteri (Fairchild \& Hertig 1953) (2.9\%) y Lu. cruciata (Coquillett 1907) (95.1\%). La presencia de esta última

Cuadro 1. Porcentaje de especies capturadas por localidad y su infección con Wolbachia.

\begin{tabular}{lccccc}
\hline \multicolumn{1}{c}{ Localidad } & Altitud msnm & Especie & $\mathrm{n}$ & $\%$ & $\begin{array}{c}\text { Individuos } \\
\text { infectados con } \\
\text { Wolbachia (\%) }\end{array}$ \\
\hline Teotihuacán del Valle & 434 & Lu. cruciata & 17 & 100 & - \\
Argovia & 608 & Lu. cruciata & 56 & 96.5 & - \\
& & Lu. carpenteri & 1 & 1.7 & - \\
& \multirow{4}{*}{662} & Lu. texana & 1 & 1.7 & - \\
San José Nexapa & Lu. cruciata & 48 & 96 & - \\
& \multirow{2}{*}{1007} & Lu. carpenteri & 2 & 4 & - \\
Guadalupe Sajú & Lu. cruciata & 75 & 92.3 & 1 - \\
& & Lu. carpenteri & 3 & 3.7 & - \\
& Lu. ovallesi & 1 & 1.2 & - \\
& Lu. shannoni & 2 & 2.5 & - \\
\hline
\end{tabular}


especie en las cuatro localidades osciló en un rango de 92.3-100\%, mientras que la presencia del resto de las especies se mantuvo en un rango de 0-4\%. Lutzomyia texana fue la única especie representada por un solo macho (Argovia), aunque otros dos machos de Lu. shannoni fueron capturados en Guadalupe Sajú. Todos los machos fueron capturados con trampas CDC. Lutzomyia cruciata y Lu. ovallesi fueron capturadas con ambos tipos de trampa, aunque sus capturas fueron más abundantes empleando la trampa Shannon. Los especímenes de $L u$. carpenteri fueron capturados empleando trampas CDC.

Amplificación de fragmentos de ADN por PCR. Los resultados de la amplificación por PCR usando los iniciadores para Leishmania no proporcionaron bandas en las muestras, solo en los controles positivos. Sin embargo, en las amplificaciones para ADN de Wolbachia encontramos un grupo positivo (Fig. 2). Los cinco individuos que formaron ese grupo fueron tamizados individualmente, resultando un individuo positivo identificado como $\mathrm{Lu}$. cruciata. Los iniciadores proporcionaron un amplicon de 900 pb correspondiente al gen 16S ADNr y 600 pb para el gen wsp (Figs. 2 y 3). Los especímenes de $\mathrm{Lu}$. cruciata fueron capturados con ambos tipos de trampas en

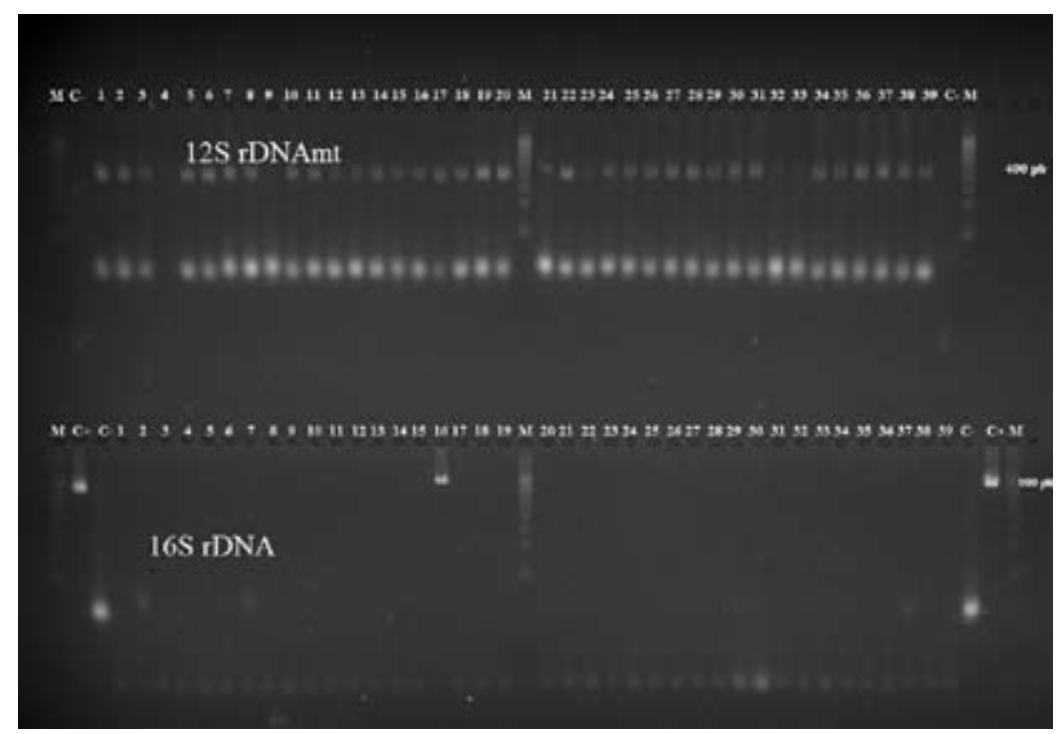

Figura 2. PCR para determinar infección por Wolbachia usando los iniciadores 76-99f y 1012-994r específicos del gen 16S ADNr (900 pb). Los iniciadores 12SAI y 12SBI específicos para el ADN mitocondrial de insecto 12S ADNr fueron usados como control de calidad de la extracción de ADN (400 pb). Líneas - M: Marcador de peso molecular; C-: control negativo; C+: control positivo; 19 muestras de San José Nexapa; 10-24 muestras de la Finca Guadalupe Zajú; 25-28 muestras de Teotihuacán del Valle; 29-39 muestras de la Finca Argovia. 


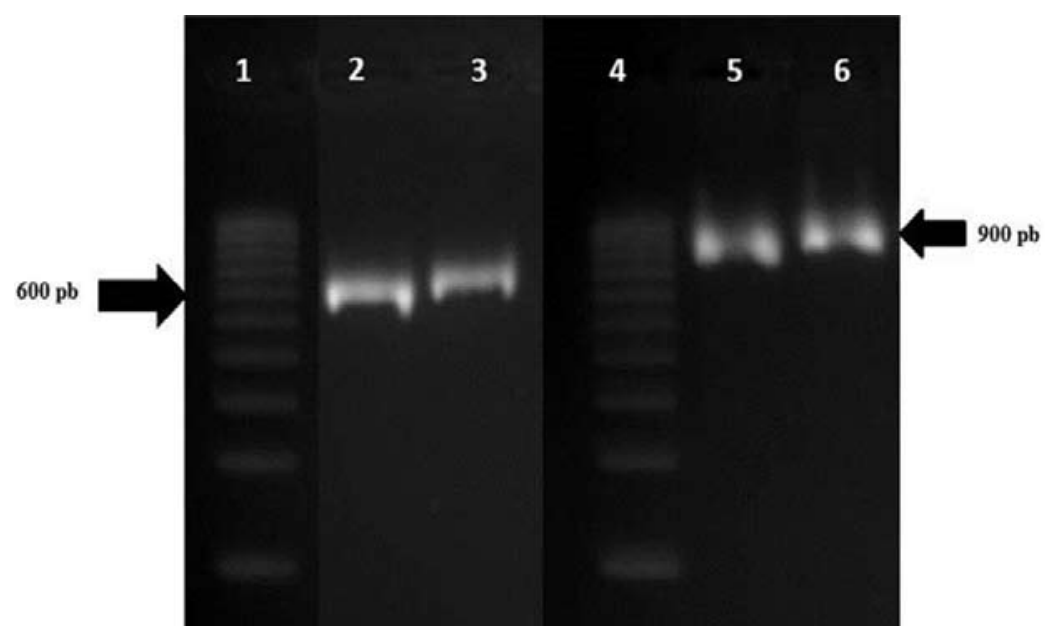

Figura 3. PCR para determinar infección por Wolbachia en el grupo 16 usando los iniciadores 76-99f y 1012-994r específicos para el gen $16 \mathrm{~S}$ ADNr (900 pb) y los iniciadores wsp 81f y wsp 691r del gen WSP. Líneas - 1,5 y 9: marcador de peso molecular; líneas - 2: C+ (control positivo) gen 16S ADNr; línea - 3: 136 (ADN Lu. cruciata) gen 16S ADNr; línea - 4: C- (control negativo) gen 16S ADNr; línea - 6: C+ (control positivo) gen WSP; línea - 7: 136 (ADN Lu. cruciata) gen WSP; línea - 8: C(control negativo) gen WSP.

tres localidades (Argovia, San José Nexapa y Guadalupe Sajú) y únicamente con trampa Shannon en Teotihuacán del Valle. En Guadalupe Sajú se capturó el espécimen infectado con Wolbachia y se identificó la mayor cantidad de especies.

\section{DISCUSIÓN}

La geografía de la Región Soconusco y sus características climáticas proporcionan ambientes ecológicos variables, que permiten albergar a un gran número de especies tanto animales como vegetales (Sánchez \& Jarquín 2004). Este estudio presenta por primera vez la composición de especies de flebótomos presentes en la Región Soconusco y documenta la presencia de las especies $L u$. texana y $L u$. carpenteri en el estado de Chiapas. Se sabía de la presencia de Lu. carpenteri en esta zona (Jeanneth Pérez, Comunicación personal), aunque no se había documentado previamente. Por su parte, las especies $\mathrm{Lu}$. shannoni, $\mathrm{Lu}$. ovallesi y $\mathrm{Lu}$. cruciata han sido reportadas para el estado de Chiapas (Ibáñez-Bernal 1999, 2002). Sin embargo, sólo Lu. cruciata está registrada para la Región Soconusco (Ibáñez-Bernal 1999). Esta especie fue predominante en todas las localidades muestreadas en el presente estudio, demostrando su amplia distribución y adaptación a esta región, ya que las especies distribuidas a lo largo de gradientes altitudinales tienden a adaptarse a una variedad de condiciones climáticas (Telfer \& Hassall 1999). Lu. carpenteri no es antropofilica y su papel en 
la transmisión de Leishmania en los animales es incierto (Rutledge et al. 1981); lo mismo ocurre con Lu. texana (Sánchez-Tejeda et al. 2001).

Entre las especies identificadas destacan algunas consideradas como vectores importantes de leishmaniasis en el sureste de México y Guatemala, como es el caso de $\mathrm{Lu}$. cruciata (Rebollar et al. 1996), Lu. shannoni (Sánchez-García et al. 2010) y Lu. ovallesi (Rowton et al. 1992). Nuestros resultados no muestran evidencias de infección en la zona de estudio. Los antecedentes clínicos de casos encontrados en la región, han sido personas que proceden de otros lugares endémicos de México o Centroamérica (Ortega-Grillasca \& Vidal-Antonio 1990). Tampoco se ha registrado infección con Leishmania en hospederos silvestres (Cigarroa 2011), lo que sugiere que la tasa de infección es muy baja y que faltan más estudios para descartar la presencia de la enfermedad en esta región de México. Sin embargo, se requieren más estudios sobre ecología de flebotominos y su posible infección con Leishmania en esta zona.

Existen antecedentes que señalan a Wolbachia como un grupo de bacterias muy abundante y con amplia distribución en artrópodos, que incluye a insectos (Werren 1997). Se ha estimado que el $20 \%$ de los insectos neotropicales podría encontrarse infectado con Wolbachia (Werren et al. 1995), aunque la presencia de este simbionte no ha sido determinada para la mayoría de las especies de insectos hasta ahora descritas. Los flebotominos forman un grupo que incluye más de 800 especies, pero solo se ha registrado infección con Wolbachia en un pequeño subgrupo de aproximadamente ocho especies (5 Lutzomyia, 3 Phlebotomus) (Azpurua et al. 2010, Cui et al. 1999, Ono et al. 2001). En este trabajo se registra por primera vez la infección natural con Wolbachia en una población silvestre de $L u$. cruciata. De las especies aquí examinadas, sólo $L u$. shannoni ha sido asociada con este simbionte a partir de especímenes recolectados en Colombia (Ono et al. 2001). En el presente estudio no fue posible registrar su presencia en $\mathrm{Lu}$. shannoni, quizá por los pocos ejemplares recolectados. Los bajos niveles de infección registrados para $L u$. cruciata y su ausencia en el resto de las especies identificadas en este trabajo sugieren que la asociación de Lutzomyia spp. con Wolbachia no es algo común en esta región. Lu. cruciata es una especie con amplia distribución y recientemente incriminada como vector de la leishmaniasis en nuestro país (Pech-May et al. 2010), por lo tanto el presente registro resulta de gran interés para el desarrollo de estrategias que permitan el manejo de Wolbachia para el control genético de los vectores de leishmaniasis. El bajo nivel de infección registrado contrasta con los descritos en otras partes del mundo para diferentes especies de flebotominos (Azpurua et al. 2010), lo que puede deberse a las bajas tasas de transmisión maternal reportadas para otras especies del grupo (Kassem \& Osman 2007). Sin embargo, la realización de estudios sobre la distribución y presencia de Wolbachia en flebominos de otras regiones de la República Mexicana y los fenotipos reproductivos que produce en ellas, son necesarios para sus futuras aplicaciones en el control de los vectores de leishmaniasis. 
Agradecimientos. Al Fondo Mixto Consejo Estatal de Ciencia y Tecnología del estado de Chiapas por financiar el proyecto "Distribución y abundancia de vectores de la Leishmaniasis en zonas cafetaleras del Soconusco” (FOMIX-77409) y al Fondo Institucional de Fomento Regional para el Desarrollo Científico, Tecnológico y de Innovación (FORDECYT) del Consejo Nacional de Ciencia y Tecnología (convenio 116306: Innovación socio-ambiental para el desarrollo en áreas de alta pobreza y biodiversidad de la frontera sur de México).

\section{LITERATURA CITADA}

Alexander, B. 1987. Dispersal of Phlebotominae sand flies (Diptera: Psychodidae) in a Colombian coffee plantation. Journal of Medical Entomology, 24: 552-558.

Alexander, B., L. A. Agudelo, F. Navarro, F. Ruiz, J. Molina, G. Aguilera \& M. L. Quiñonez. 2001. Phlebotomine sandflies and leishmaniasis risk in Colombian coffee plantations under two systems of cultivation. Medical and Veterinary Entomology, 15: 364-373.

Alexander, B., E. Barbosa, E. Haigh \& L. Leal. 2002. Transmission of Leishmania in coffee plantations of Minas Gerais, Brazil. Memórias do Instituto Oswaldo Cruz, 97: 627-630.

Alexander, B. \& M. Maroli. 2003. Control of Phlebotomine Sandflies. Medical and Veterinary Entomology, 17: 1-18.

Ashford, R.W. 2000. The leishmaniases as emerging and reemerging zoonoses. Journal of Parasitology, 30: 1269-1281.

Azpurua, J., D. De La Cruz, A. Valderama \& D. Windsor. 2010. Lutzomyia Sand Fly Diversity and Rates of Infection by Wolbachia and an Exotic Leishmania Species on Barro Colorado Island, Panama. PLoS Neglected Tropical Diseases, 4(3): e627.

Becker, I., G. Carrada-Figueroa, M. Gudiño-Zayas, C. González, Berzunza-Cruz M, B. RivasSánchez \& O. Velasco-Castrejón. 2005. Análisis de la leishmaniasis en México, pp. 27-33. Consulta de expertos OPS/OMS sobre la leishmaniasis visceral en las Américas. Informe Final. Brasilia, Brasil. Noviembre del 23-25.

Chongo, A. M. L. \& E. R. García. 2010. Leishmaniasis y transfusión. Revista Mexicana de Medicina y Transfusión, 3 (Supl. 1): S42-S47.

Cigarroa, T. N. 2011. Detección de reservorios de Leishmaniasis cutánea en mamíferos silvestres y domésticos en la región Fronteriza de Chiapas, México. Tesis de Licenciatura, Universidad Autónoma de Chiapas, Centro de Biociencias, México.

Cui, L., S. H. Chang, D. Strickman \& E. Rowton. 1999. Frequency of Wolbachia Infection in Laboratory and Field Sand Fly (Diptera:Psychodidae) Populations. Journal of America Mosquito Control Association, 15: 571-572.

Glaser, R. L. \& M. A. Meola. 2010. The Native Wolbachia Endosymbionts of Drosophila melanogaster and Culex quinquefasciatus Increase Host Resistance to West Nile Virus Infection. PLoS ONE. 5(8): e11977.

Hilgenboecker, K., P. Hammerstein, P. Schlattmann, A. Telschow \& J. H. Werren. 2008. How many species are infected with Wolbachia? - a statistical analysis of current data. FEMS Microbiology Letter, 281: 215-220.

Hoffmann, A. A., B. L. Montgomery, J. Popovici, I. Iturbe-Ormaetxe, P. H. Johnson, F. Muzzi, M. Greenfield, M. Durkan, Y. S. Leong, Y. Dong, H. Cook, J. Axford, A. G. Callahan, N. Kenny, C. Omodei, E. A. McGraw, P. A. Ryan, S. A. Ritchie, M. Turelli \& S. L. O’Neill. 2011. Successful establishment of Wolbachia in Aedes populations to suppress dengue transmission. Nature, 476: 454-457. 
Ibáñez-Bernal, S. 1999. Phlebotominae (Diptera: Psychodidae) de México. I.- Bruptomyia Franca y Parrot; Lutzomyia França, las especies de L. (Lutzomyia) Franca y del grupo Verrucarum. Folia Entomológica Mexicana, 107: 61-116.

Ibáñez-Bernal, S. 2001. Phlebotominae (Diptera: Psychodidae) de México. II.- Las especies de Lutzomyia (Coromyia) Barreto, del grupo Delpazoi y de Lutzomyia (Dampfomyia) Addis. Folia Entomológica Mexicana, 40: 17-46.

Ibáñez-Bernal, S. 2002. Phlebotominae (Diptera: Psychodidae) de México. III. -Las especies de Lutzomyia (Psathyromyia) Barreto, del grupo Aragoi, de L. (Trichopygomyia) Barreto, del grupo Dreisbachi y de L. (Nyssomyia) Barreto. Folia Entomológica Mexicana, 41: 149-183.

Ibáñez-Bernal, S. 2003. Phlebotominae (Diptera: Psychodidae) de México. IV.- Las especies de Lutzomyia (Psychodopygus) Mangabeira, L. (Micropygomyia) Barreto, Lutzomyia grupo oswaldo, L. (Helicocyrtomyia) Barreto y especies del género sin agrupar. Folia Entomológica Mexicana, 42: 109-152.

Ibáñez-Bernal, S. 2005a. Phlebotominae (Diptera: Psychodidae) de México. V.- Clave ilustrada para la identificación de los machos de Lutzomyia França. Folia Entomológica Mexicana, 44(1): 49-66.

Ibáñez-Bernal, S. 2005b. Phlebotominae (Diptera: Psychodidae) de México. VI.- Clave ilustrada para la identificación de las hembras de Lutzomyia França. Folia Entomológica Mexicana, 44(2): 195212.

Ibáñez-Bernal, S., R. Hernández \& F. Mendoza. 2006. Collections of Bruchomyiinae and Phlebotominae (Diptera: Psychodidae) from the north-central portion of the state of Veracruz, Mexico, with the description of a new species. Zootaxa, 1270: 19-33.

Ibáñez-Bernal, S., E. May-Uc \& E. A. Rebollar-Tellez. 2010. Two new species of phlebotomine sand flies (Diptera: Psychodidae, Phlebotominae) from Quintana Roo, Mexico. Zootaxa, 2448: 26-34.

Ibáñez-Bernal, S., G. Rodríguez-Domínguez, C. H. Gómez-Hernández \& J. R. Ricardez-Esquinca. 2004. First Record of Lutzomyia evansi (Nuñez-Tovar 1924) in Mexico (Diptera: Psychodidae, Phlebotominae). Memórias do Instituto Oswaldo Cruz, 99: 127-129.

Ibáñez-Bernal, S., M. T. Suárez-Landa \& F. Mendoza. 2011. An updated checklist of the phlebotomine sandflies of Veracruz, Mexico (Diptera: Psychodidae, Phlebotominae). Zootaxa, 2928: 29-40.

Kassem, H. A. \& G, Osman. 2007. Maternal transmission of Wolbachia in Phlebotomus papatasi (Scopoli). Annals of Tropical Medicine and Parasitology, 101: 435-440.

Kato, H., H. Uezato, K. Katakura, M. Calvopiña, J. D. Marco, P. A. Barroso, E. A. Gomes, T. Mimori, M. Korenaga, H. Iwata, S. Nonaka \& Y. Hashiguchi. 2005. Detection and identification of Leishmania species within naturally infected sand flies in the Andean areas of Ecuador by polymerase chain reaction. American Journal of Tropical Medicine and Hygiene, 72: 87-93.

Montero-Pau, J., A. Gómez \& J. Muñoz. 2008. Application of an inexpensive and high-throughput genomic DNA extraction method for the molecular ecology of zooplanktonic diapausing eggs. Limnology and Oceanography: Methods, 6: 218-222.

Ono, M., H. R. Braig, L. E. Munstermann, C. Ferro \& S. L. O’Neill. 2001. Wolbachia Infections of Phlebotomine Sand Flies (Diptera: Psychodidae). Journal of Medical Entomology, 38: 237-241.

O’Neill, S. L., R. Giordano, A. M. E. Colbert, T. L. Karr \& H. M. Robertson. 1992. 16S rRNA phylogenetic analysis of the bacterial endosymbionts associated with cytoplasmic incompatibility in insects. Proceedings of the National Academy of Sciences of the United States of America, 89: 2699-2702.

Ortega-Grillasca, F. \& E. Vidal-Antonio. 1990. Prevalencia de leishmaniasis en tres localidades del Soconusco Chiapas (Tuxtla Chico, Tapachula y Escuintla). Tesis de Licenciatura, Universidad Nacional Autónoma de Chiapas, Ciencias Químicas, México. 
Pech-May, A., F. J. Escobedo-Ortegón, M. Berzunza-Cruz \& E. A. Rebollar-Téllez. 2010. Incrimination of four sandfly species previously unrecognized as vectors of Leishmania parasites in Mexico. Medical and Veterinary Entomology, 24: 150-161.

Rebollar-Tellez, E. A., F. Reyes-Villanueva, I. Fernandez-Salas \& F. Andrade-Narvaez.1996. Population Dynamics and Biting Rhythm of the Anthropophilic Sandfly Lutzomyia cruciata (Diptera: Psychodidae) in Southeast, Mexico. Revista do Instituto de Medicina Tropical de São Paulo, 38: 29-33.

Remme, J. H. F., E. Blas, L. Chitsulo, P. M. P. Desjeux, H. D. Engers, T. P. Kanyok, J. F. K. Kayondo, D. W. Kioy, V. Kumaraswami, J. K. Lazdins, P. P. Nunn, A. Oduola, R. G. Ridley, Y. T. Toure, F. Zicker \& C. M. Morel. 2002. Strategic emphases for tropical diseases research: a TDR perspective. Trends in Parasitology, 18(10):421-426.

Rowton, E. D., M. De Mata, N. Rizzo, C. H. Porter \& T. R. Navin. 1992. Isolation of Leishmania braziliensis from Lutzomyia ovallesi (Diptera:Psychodidae) in Guatemala. American Journal of Tropical Medicine and Hygiene, 46: 465-468.

Rutledge, L. C., B. C. Walton \& D. A. Ellenwood. 1981. A transect study of sand fly populations in Panama (Diptera: Psychodidae). Environmental Entomology, 5: 1149-1154.

Sánchez-García, L., M. Berzunza-Cruza, I. Becker-Fausera \& E. A. Rebollar-Téllez. 2010. Sand flies naturally infected by Leishmania (L.) mexicana in the peri-urban area of Chetumal city, Quintana Roo, México. Transactions of the Royal Society of Tropical Medicine and Hygiene, 104: 406411.

Sánchez, J. E. \& R. Jarquín. 2004. La Frontera Sur. Reflexiones sobre el Soconusco, Chiapas y sus problemas ambientales, poblacionales y productivos. Comisión de Asuntos Fronterizos Sur, Mexico, $300 \mathrm{pp}$.

Sánchez-Tejeda, G., N. Rodríguez, C. I. Parra, O. Hernandez-Montes, D. C. Barker \& A. MonroyOstria. 2001. Cutaneus leishmaniasis caused by members of Leishmania braziliensis complex in Nayarit, state of Mexico. Memórias do Instituto Oswaldo Cruz, 96: 15-19.

Scorza, J. V., L. Castillo, S. Rezzano, M. Márquez \& J. C. Márquez. 1985. El papel del cafeto en la endemicidad de la leishmaniasis cutánea en Venezuela. Boletín de Malariología y Salud Ambiental, 25: 82-87.

Shannon, R. 1939. Methods for collecting and feeding mosquitos in jungle yellow fever studies. American Journal of Tropical Medicine, 19: 131-148.

Sinkins, S. P. \& H. C. Godfray. 2004. Use of Wolbachia to drive nuclear transgenes through insect populations. Proceedings of the Royal Society of London. Series B, 271: 1421-1426.

Sudia, W. D. \& R. W. Chamberlain. 1962. Battery-operated light trap, an improved model. Mosquito News, 22: 126-129.

Telfer, G. T. \& M. Hassall. 1999. Ecotypic differentiation in the grasshopper Chorthippus brunneus: life history varies in relation to climate. Oecologia, 121: 245-254.

Walker, T., P. H. Johnson, L. A. Moreira, I. Iturbe-Ormaetxe, F. D. Frentiu, C. J. McMeniman, Y. S. Leong, Y. Dong, J. Axford, P. Kriesner, A. L. Lloyd, S. A. Ritchie, S. L. O’Neill \& A. A. Hoffmann. 2011. The wMel Wolbachia strain blocks dengue and invades caged Aedes aegypti populations. Nature, 476: 450-455.

Werren, J. H., D. Windsor \& L. Guo. 1995. Distribution of Wolbachia among neotropical arthropods. Proceedings of the Royal Society of London. Series B, 262:197-204.

Werren, J. H. 1997. Biology of Wolbachia. Annual Review of Entomology, 42: 587-609.

Werren, J. H., L. Baldo \& M. E. Clark. 2008. Wolbachia: master manipulators of invertebrate biology. Nature Reviews Microbiology, 6: 741-751. 
World Health Organization (WHO). Technical report series no. 949. Control of the leishmaniasis: report of a meeting of the WHO Expert Committee on the Control of Leishmaniases, Geneva, 22-26 March 2010.

Young, D. A. \& M. A. Duncan. 1994. Guide to the identification and geographic distribution of Lutzomyia sand flies in Mexico, the West Indies, Central and South America (Diptera: Psychodidae). Memoirs of the American Entomological Institute, 54: 1-881.

Zhou, W., F. Rousset \& S. L. O’Neill. 1998. Phylogeny and PCR-based classification of Wolbachia strains using wsp gene sequences. Proceedings of the Royal Society of London, Series B, 265: 509515. 